\title{
Reduction of Intramuscular Nerve Branching and Synaptogenesis Is Correlated with Decreased Motoneuron Survival
}

\author{
Jicheng Tang and Lynn Landmesser \\ Department of Physiology and Neurobiology, The University of Connecticut, Storrs, Connecticut 06269-3042
}

Blockade of neuromuscular activity during the period of naturally occurring cell death increases intramuscular nerve branching, synaptogenesis, and survival of embryonic chicken motoneurons. These results suggested that the enhanced motoneuron survival observed might result not from increased production of a trophic factor by the target, as often suggested, but rather from the enhanced ability of motoneurons to take up this factor via additional branches and/ or synapses. Since removal of polysialic acid (PSA) by PSAspecific endoneuraminidase (endo $N$ ) during the period of nerve ingrowth and motoneuron cell death had been shown to reduce intramuscular nerve branching by altering adhesive interactions between axons and myotubes, we wished to determine, first, if this resulted in a reduction of synapse formation, and second, if this was correlated with decreased motoneuron survival. When PSA was removed from embryonic day $6(E 6)$ to $E 9$ by injecting endo $N$ into the limb, the number of synapses in the posterior iliotibialis muscle was greatly reduced both at E6.5, the onset of the cell death period, and at E7.5-E8, the middle of the cell death period. Total motoneuron counts at both E7.5-E8 and E10 (close to the end of cell death period) were significantly decreased compared to controls. These results indicate that a treatment that reduces intramuscular nerve branching and synapse formation also affects motoneuron survival. Furthermore, since removal of PSA in ovo via endo $\mathrm{N}$ altered neither the spontaneous embryonic motility nor the activation pattern of motoneurons or muscles, these experiments avoid the complication of activity blockade that may alter motoneuron survival by affecting events other than, or in addition to, intramuscular nerve branching.

[Key words: polysialic acid, nerve branching, synaptogenesis, neuronal death, trophic factors, activity]

Target-derived trophic molecules have been considered to play a crucial role in allowing neurons to survive during the period of naturally occurring neuronal cell death (for review, see Oppenheim 1991). For chick motoneurons, chronic blockade of neuromuscular activity with $d$-tubocurarine (dTC) or $\alpha$-bun-

\footnotetext{
Received Nov. 4, 1992; revised Jan. 20, 1993; accepted Feb. 10, 1993.

We thank Liviu Cupceancu for his technical assistance, Karen Sommer for typing the manuscript, Dr. Urs Rutishauser for supplying endo N, and Dr. Kathleen Buckley for supplying SV2 antibodies. This work was supported by NIH Grant NS 19640 and a University of Connecticut predoctoral fellowship to Jicheng Tang.

Correspondence should be addressed to Dr. Lynn Landmesser, Department of Physiology and Neurobiology, The University of Connecticut, 75 North Eagleville Road, Box U-42, Storrs, Connecticut 06269.

Copyright (C) 1993 Society for Neuroscience $0270-6474 / 93 / 133095-09 \$ 05.00 / 0$
}

garotoxin ( $\alpha$-BTX) during the normal neuronal death period rescued almost all of the spinal motoneurons that would normally die (Pittman and Oppenheim, 1978, 1979). Although the proposed trophic molecule(s) for motoneurons has yet to be identified and definitive proof for the trophic hypothesis as related to naturally occurring cell death is still lacking, two alternative ideas have been proposed to explain how activity blockade may affect motoneuron survival. The first is that a target-derived trophic factor is normally limited in amount and that activity blockade results in an upregulation of this trophic factor(s). This idea was supported by studies of denervated or inactive adult muscle (Henderson et al., 1983; Hill and Bennett, 1983), but not of embryonic muscle. In the latter case, an in vitro study (Tanaka, 1987) revealed that blockade of embryonic neuromuscular transmission did not increase the motoneuron survival-promoting activity of extracts prepared from such muscles. When extracts from activity-blocked muscles were tested in vivo for motoneuron survival promoting activity, they were no more effective than extracts prepared from normal muscles.

An alternative explanation for the effect of neuromuscular activity blockade on motoneuron survival would be that the amount of trophic factor normally available from target muscles might be limited, not by the amount of trophic factor provided by the muscles, but by access (Meriney and Pilar, 1987; Dahm and Landmesser, 1988; Oppenheim, 1989). In other words, the sites where motoneurons are able to take up these factors might be limited. It has been postulated that such access sites might be synaptic sites or nerve endings on myotubes. Therefore, any perturbations that alter axonal branching and synaptogenesis would be expected to change the number of access sites and affect motoneuron survival. Some early reports indicated that at later embryonic stages there was an increase in the density of innervation in dTC- or $\alpha$-BTX-blocked muscle (Pittman and Oppenheim, 1979; Ding et al., 1983; Oppenheim and Chu-Wang, 1983). A more detailed examination of intramuscular nerve branching and synapse formation following activity blockade during the period of naturally occurring cell death (Dahm and Landmesser, 1988, 1991) found a greatly increased number of nerve branches and synapses. Since the peak rate of increased nerve branching and synaptogenesis occurred prior to the onset of motoneuron cell death, alteration of the number of nerve branches and synapses was suggested to be the cause of, rather than a result of, the increased motoneuron survival (Dahm and Landmesser, 1991). However, activity blockade is complicated in that it may alter motoneuron survival by affecting events other than, or in addition to, intramuscular nerve branching and synaptogenesis. For this reason it was important to develop a way of altering intramuscular nerve branching and synapse formation without affecting activity. 
Recently, it was found that intramuscular nerve branching could be modified by altering the function of adhesion molecules such as NCAM and Ll by in ovo, intramuscular injection of specific functional blocking antibodies (Landmesser et al., 1988). Anti-NCAM reduced intramuscular branching while anti-L1 increased it. Although these perturbations did not alter neuromuscular activity, it was difficult to maintain in ovo blocking concentrations of such antibodies during the entire period of intramuscular nerve branching and motoneuron cell death. Second, the effects of antibody injection were localized to the several injected muscles such that it would have been necessary to backlabel these individual motoneuron pools to determine if survival had been affected. Third, perturbation of NCAM or L1 function, while altering branching, may not reflect the normal developmental regulation of such cell adhesion molecules. For example, during the period of intramuscular nerve branching and motoneuron cell death in normal embryos, we did not detect changes in L1 or NCAM levels. Similarly, we did not detect any changes in the levels of these molecules following activity blockade even though the degree of fasciculation and the number of nerve branches changed (Landmesser et al., 1988, 1990). However, we found that polysialic acid (PSA), a posttranslational carbohydrate modification of NCAM, was dramatically increased following activity blockade (Landmcsscr ct al., 1990).

It has become increasingly appreciated that PSA on NCAM can modulate not only the function of NCAM (Hoffman and Edelman, 1983; Doherty et al., 1990), but also the function of other cell adhesion molecules such as L1 and laminin (Rutishauser et al., 1988; Landmesser et al., 1990; Acheson et al., 1991), and that the level of PSA is developmentally regulated (Chuong and Edelman, 1984; Sunshine et al., 1987; Landmesser et al., 1990; Tang et al., 1992). Since removal of PSA by a PSAspecific endoneuraminidase (endo $N$ ) during the period of nerve ingrowth and motoneuron cell death reduced intramuscular nerve branching both in normal embryos and in those subjected to activity blockade (Landmesser et al., 1988, 1990), we wished to determine, first, if this resulted in a reduction in synapse formation, and second, if this was correlated with decreased motoneuron survival. When PSA was removed throughout the period of intramuscular nerve branching and synaptogenesis by in ovo injections of endo $\mathrm{N}$ into the limb, the number of synapses in the posterior iliotibialis muscle (PITIB) was greatly reduced as was motoneuron survival. Since removal of PSA altered neither spontaneous embryonic motility nor the activation pattern of motoneurons or muscles assessed electrophysiologically, these experiments avoid the complication of activity blockade that may alter motoneuron survival by affecting events other than, or in addition to, intramuscular nerve branching and synapse formation.

This work has been reported briefly in abstract form (Tang and Landmesser, 1992).

\section{Materials and Methods}

In ovo injection of endo $N$. As previously described (Landmesser et al., 1990; Tang et al., 1992), the purified endo $N$ used to remove PSA in this study has been well characterized and has a strict specificity for $\alpha-2,8$-linked PSA of chain length greater than five sialyl residues and a neutral pH optimum (Vimr et al., 1984; Rutishauser et al., 1985; Acheson et al., 1991)

White Leghorn chick embryos were raised in a forced-draft incubator, and at appropriate stages holes were made in the shells and a coverslip was sealed in place with melted paraffin (Landmesser et al., 1990; Tang et al., 1992). Between 0.1 and $1.0 \mu \mathrm{l}$ of a solution containing 30,000 $\mathrm{U} / \mathrm{ml}$ of endo $\mathrm{N}$ was injected into the limb of embryos at stages (St)
28-29 (Hamburger and Hamilton, 1951) [ embryonic day 5.5 (E5.5) to E6, the onset of cell death period]. Previous results showed that this treatment removed all PSA [detected by the PSA-specific monoclonal antibody (mAb) 5A5] in frozen sections from the injected limbs from several hours after injection (the earliest time tested) up to $4 \mathrm{~d}$, but had no effect on NCAM levels, as visualized with $\mathrm{mAb} 5 \mathrm{E}$ (Landmesser et al., 1990; Tang et al., 1992). Thus, the treatment performed in the present experiments would have removed PSA throughout most of the period of nerve branching, synapse formation, and motoneuron cell death (St 29-36; Dahm and Landmesser, 1988, 1991).

Muscle whole-mounts. Control and endo N-treated embryos were decapitated, eviscerated, and placed in oxygenated Tyrode's at room temperature $\left(20-23^{\circ} \mathrm{C}\right)$. Skin and connective tissue were removed to reveal the underlying muscles. The posterior iliotibialis (PITIB) muscle was carefully stripped of all connective tissue, but left attached to the limb during all staining procedures (Dahm and Landmesser, 1988, 1991).

To visualize intramuscular nerve branching, muscle whole-mounts were stained with $\mathrm{C}-2$, a mouse $\mathrm{IgG}$ monoclonal antibody against a neuronal cytoskeletal element (Dahm and Landmesser, 1988). Briefly, muscles were fixed in cold acetone for $2 \mathrm{~min}$, washed in PBS, incubated in C-2 (undiluted hybridoma supernatant) containing $0.3 \%$ Triton for $1 \mathrm{hr}$ at room temperature, washed in PBS, fixed in 3.7\% formaldehydePBS for $15 \mathrm{~min}$, washed in PBS, incubated in the appropriate fluorescein isothiocyanate (FITC)-labeled secondary antibody for $1 \mathrm{hr}$ at room temperature or overnight at $4^{\circ} \mathrm{C}$, and washed in PBS. The muscles were removed from the limb and mounted between two coverslips in $50 \%$ glycerin-PBS containing $0.3 \mathrm{mg} / \mathrm{ml} p$-phenylenediamine (Sigma) to prevent fading.

Synapse quantification. In this study, a synapse was defined as the colocalization of a presynaptic profile containing SV2 (as visualized with a mouse IgG monoclonal that recognizes a synaptic vesicle antigen, Buckley and Kelly, 1985), and an acetylcholine receptor (AChR) cluster labeled with rhodamine- $\alpha$-bungarotoxin (R-BTX) (Dahm and Landmesser, 1991).

To visualize the synapses, $15 \mu \mathrm{m}$ cryostat sections were made from limbs that had been fixed for $1 \mathrm{hr}$ in $3.7 \%$ formaldehyde-PBS and cryoprotected by incubating overnight in $30 \%$ sucrose prior to freezing. Sections were stained with SV2 and R-BTX for $2 \mathrm{hr}$, rinsed in PBS, stained with the secondary antibody anti-mouse IgG FITC for $1 \mathrm{hr}$, rinsed, and mounted in $50 \%$ glycerin-PBS containing $0.3 \mathrm{mg} / \mathrm{ml} p$-phenylenediamine. The total number of synapses was counted in the PITIB muscle at St 30 and St 33 from every fourth or fifth section, respectively. Synapses were counted, as in our previous study (Dahm and Landmesser, 1991), directly under microscopic visualization (Nikon Microphot at $400 \times$ magnification). Data were obtained in this way for both control and endo $\mathrm{N}$-treated embryos.

We counted both the total number of $\mathrm{AChR}$ clusters and those that were colocalized with SV2-containing profiles (by our definition, synapses). The data we obtained for both parameters agreed well with that of the previous study at both St 30 and St 33 (present data presented first): synapses at St 30,1627 versus 2223 , and at St $33,8558 \pm 783$ versus $7725 \pm 1367$; total clusters at $S t 30,4952 \pm 101$ versus 5810 , and at St 33, 19,651 \pm 1442 versus $16,747 \pm 4600$.

Since in the chick the intramuscular nerves initially induce $\mathrm{AChR}$ clusters, presumably via release of a diffusible clustering factor, all along their length and up to several hundred micrometers (four or five myotube clusters) away (Dahm and Landmesser, 1991), many clusters at St 30 and St 33 are not colocalized with SV2-containing profiles. It is only by St 36 that most of the noncolocalized receptors are lost and colocalization approaches $100 \%$. Once again the value for colocalization we obtained at St 33,44\%, was similar to that obtained by Dahm and Landmesser (1991), which was $46 \%$. We chose to count clusters and synapses directly via microscopic visualization, rather than by overlaying negatives, since we could for each cluster scan back and forth through the entire focal plane contained within the $15 \mu \mathrm{m}$ section and be certain that a given cluster was actually in contact (over some portion of its length) with an SV2-containing profile. This was especially necessary at these developmental stages since the SV2-containing presynaptic terminal does not occupy the entire length of the postsynaptic AChR cluster (see Dahm and Landmesser, 1991, for additional details). When comparing both methods previously (Dahm and Landmesser, 1991), we found that this gave us more consistent results, and that the photographic overlay method resulted in a number of synapses being missed.

When quantifying the total number of synapses in an entire PITIB muscle, errors could result from counting the same synapse in each of two adjacent sections. To determine if corrections for double counting 

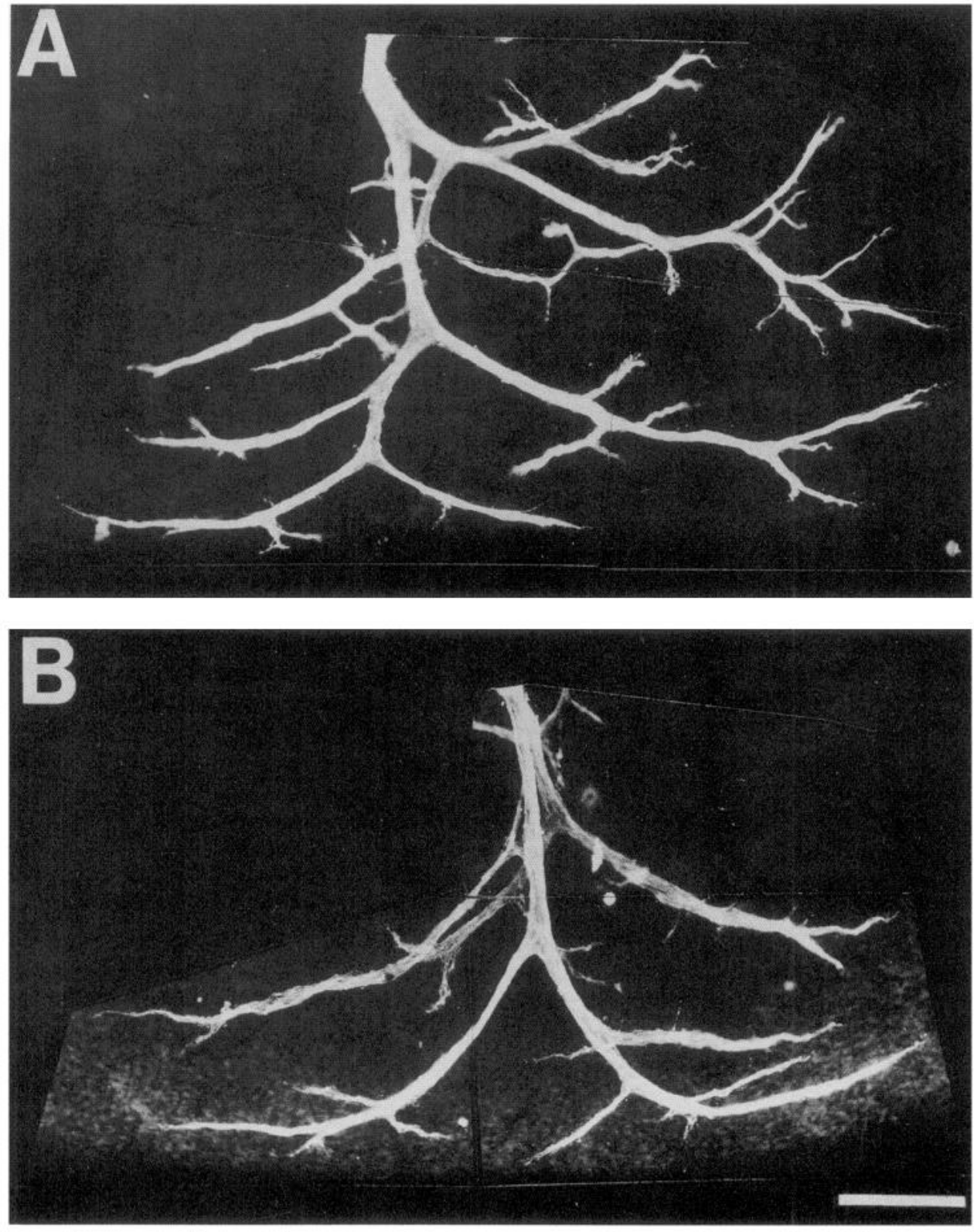

Figure 1. The effect of PSA removal on intramuscular nerve branching in the PITIB muscle. $A$, PITIB muscle from a control embryo at St 32. The entire nerve branching pattern was revealed by immunostaining with the neuronspecific antibody C2. $B$, PITIB muscle from an endo $\mathrm{N}$-treated embryo at same stage. Although the main intramuscular trunks grow in a similar way as controls, removal of PSA results in a decrease in the number of branch points and thus a decrease in the number of total branches. Scale bar, $200 \mu \mathrm{m}$. were required, the length of synapses was measured from muscle wholemounts where the entire length of synapses could be visualized at St 30 and St 33 in both control and endo $\mathrm{N}$-treated muscles (the same staining protocol was used for frozen sections except that $0.3 \%$ Triton was added to the primary and secondary antibodies). The mean length of synapses did not differ between control and endo $\mathrm{N}$-treated muscles at St 30 (3.9 \pm 2.0 and $4.1 \pm 2.4 \mu \mathrm{m}$, mean $\pm \mathrm{SD}$, respectively) or at St $33(5.2 \pm$ 2.6 and $5.9 \pm 3.7 \mu \mathrm{m}$, mean $\pm \mathrm{SD}$, respectively). Thus, any corrections for double counting would have reduced the control and the endo $\mathrm{N}$ values to the same extent, and these were not performed.

Motoneuron quantification. The lumbosacral spinal cords from both control and endo N-treated embryos were fixed in Bouin's solution, dehydrated in a graded series of ethanol and xylene, and embedded in paraffin. Serial $10-\mu \mathrm{m}$-thick sections were stained with hematoxylin and eosin-orange $\mathrm{G}$ (Tanaka and Landmesser, 1986). Counts were made on every 5th (St 33 and St 36) or 10th (St 39) section at $400 \times$ throughout the lumbosacral enlargement. All large cell profiles that contained a nucleus and were located in the lateral motor column (LMC) were counted. Nuclear diameters were measured and no significant differences were found between control and endo $\mathrm{N}$-treated embryos at $\mathrm{St}$ $33(8.08 \pm 0.94$ and $8.56 \pm 1.27 \mu \mathrm{m}$, mean $\pm \mathrm{SD}$, respectively) or at St $36(9.36 \pm 1.08$ and $9.05 \pm 0.86 \mu \mathrm{m}$, mean $\pm \mathrm{SD}$, respectively). Thus, no corrections for double counting (e.g., see Abercrombie, 1946) were made, and raw counts are presented in the tables.
Electrophysiology. As described previously (Landmesser and O'Donovan, 1984a; Landmesser and Szente, 1986), embryos from St 34-36 (control or endo $\mathrm{N}$ treated) were quickly decapitated and eviscerated, and a ventral laminectomy was performed. Isolated spinal cord-hindlimb preparations were rapidly placed in well-oxygenated Tyrode's solution at $30^{\circ} \mathrm{C}$ for several hours, after which time the embryos become spontaneously active. We characterized motoneuron activation patterns by making electromyographic (EMG) recordings with suction electrodes from pairs of hindlimb muscles (usually one flexor and one extensor) following single stimuli to the thoracic or cervical cord at $10 \mathrm{~min}$ intervals. The activation patterns were amplified and filtered $(3 \mathrm{~Hz}$ to 3 $\mathrm{kHz}$ ) and displayed on line on a Gould chart recorder in addition to being recorded on a Vetter Instrumentation tape recorder for subsequent analysis.

\section{Results}

Reduction of intramuscular nerve branching following removal of PSA is correlated with decreased synapse formation

Previous studies showed that removal of PSA during the period of intramuscular nerve branching resulted in a reduction in the number of side branches in the iliofibularis (IFIB) muscle both in normal and in dTC-treated embryos (Landmesser et al., 1988, 

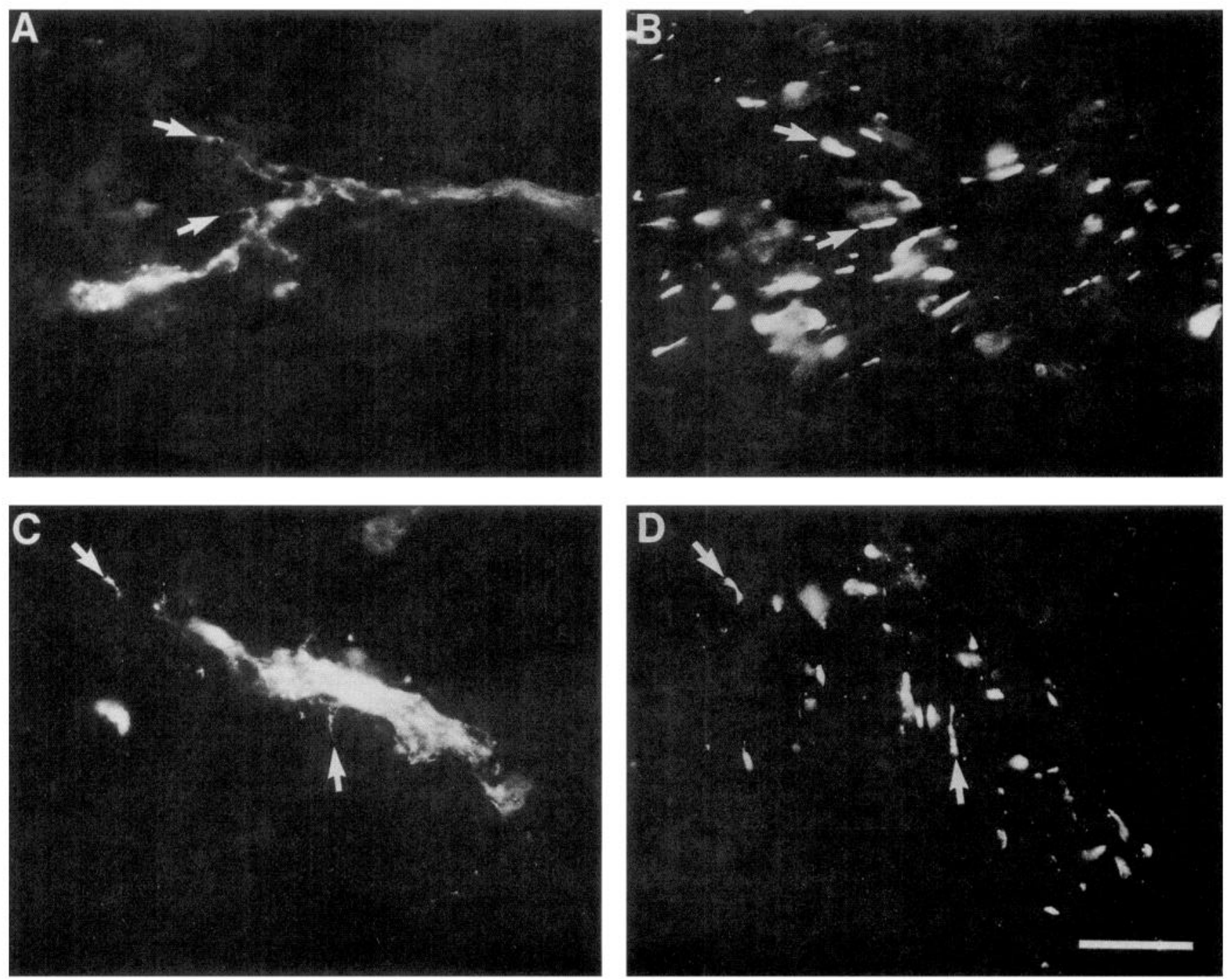

Figure 2. The distribution of SV2- and R-BTX-labeled profiles in frozen cross sections of the PITIB muscle in normal $(A, B)$ and endo N-treated $(C, D)$ embryos at St 33. Axons are labeled with SV2 $(A, C)$ and AChR clusters are labeled with R-BTX $(B, D)$ in the same sections. Arrows indicate several nerve profiles $(A, C)$ and corresponding colocalized AChR clusters $(B, D)$. Scale bar, $25 \mu \mathrm{m}$.

1990). To avoid the complication that the IFIB is partially slow and slow myotubes have multiple synapses, we chose to quantify synapses in the all fast PITIB muscle. Since we wanted to use the PITIB muscle to determine if a change in nerve branching results in a change in synapse formation, we needed first to show if branching had also been reduced in the PITIB muscle, similar to the IFIB muscle for which we had previously obtained quantitative data (Landmesser et al., 1990). As shown in Figure 1, a single injection of endo $\mathrm{N}$ at St 28 (E5.5) resulted in a decrease in the number of points of bifurcation and thus a decrease in the total number of branches in the PITIB muscle (compare Fig. $1 A, B)$.

To determine if this reduction in branching was correlated with decreased synapse formation, we counted synapses from immunostained frozen cross sections of the PITIB muscle, both in normal embryos and in those subjected to endo $\mathrm{N}$ treatment. A synapse was defined as previously (Dahm and Landmesser, 1991) by the colocalization of SV2, a synaptic vesicle antigen (Buckley and Kelly, 1985), and R-BTX to label postsynaptic AChR clusters (see Materials and Methods for additional details). As shown in Figure 2 and described previously (Dahm and Landmesser, 1991) at early stages of chick muscle innervation (St 30-34) the intramuscular nerve branches induce clusters of AChRs all along their length and on myotubes up to several hundreds of micrometers distant, presumably by release of diffusible clustering factors. At St 33 (Fig. $2 B$ ) only about $40 \%$ of these clusters are colocalized with a presynaptic profile. In the single focal plane shown in this figure, several clusters that are colocalized with SV2 stained profiles are indicated by arrows (Fig. 2A). In endo $\mathrm{N}$-treated embryos, the nerves also induced many noncolocalized clusters and the percentage of colocalization at this stage $(32 \%)$ was similar to control values (44\%) and to previously published control values (46\%; Dahm and Landmesser, 1991). Colocalized clusters in both endo Ntreated and control embryos were similar in appearance and their overall length did not differ; at St 30 it was $3.9 \pm 2.0$ versus $4.1 \pm 2.4 \mu \mathrm{m}$ for control and endo $\mathrm{N}$-treated embryos, respectively. By St 33, the length of synapses had increased slightly but still did not differ significantly between control (5.2 $\pm 2.6 \mu \mathrm{m})$ and endo $\mathrm{N}$-treated $(5.9 \pm 3.7 \mu \mathrm{m}$, mean $\pm \mathrm{SD})$ embryos.

We next counted the total number of synapses in the entire 


\section{$\square$ Control}

$\square$ Endo $\mathrm{N}$

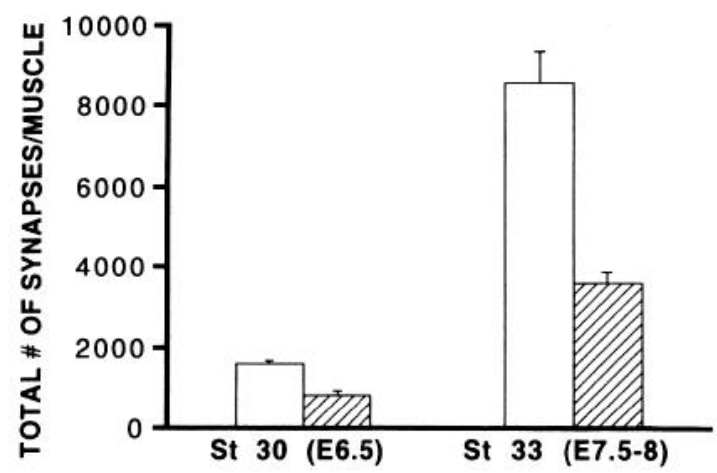

Figure 3. The number of synapses in the PITIB muscle during normal development and following PSA removal. The data are from St 30 and St 33 controls (open bar) or endo $\mathrm{N}$-injected embryos (hatched bar). Error bars represent $\mathrm{SD} ; n=3$. The number of synapses following endo $\mathrm{N}$ injection at St 28 is significantly decreased at both St 30, the onset of cell death period, and at St 33 , the middle of cell death period $(P \leq$ $0.001)$.

PITIB muscle at St 30 (E6.5, the onset of cell death period) and St 33 (E7.5-E8, the middle of cell death period) both in control embryos and in those treated with endo $\mathrm{N}$. The results are presented in Figure 3. Removing PSA reduced the number of synapses in the PITIB muscle to approximately $50 \%$ of control values at St 30 (E6.5) and to approximately $40 \%$ of control values at St 33 (E7.5-8). Thus, the reduction of intramuscular nerve branching produced by removal of PSA was correlated with a reduction in synapse formation.

\section{Reduced branching and synaptogenesis are correlated with decreased motoneuron survival}

To determine if this treatment that reduced branching and synapse formation was correlated with altered motoneuron survival, motoneuron counts were performed at both St 33 (E7.5E8), the middle of cell death period, and St 36 (E10), the end of cell death period. Since a single injection of endo N at St 28 29 (E5-E6) removed PSA from the entire injected limb for $4 \mathrm{~d}$ (Landmesser et al., 1990), we assumed that branching and synapse formation would be reduced in other limb muscles in a similar fashion to the PITIB muscle that we had quantified. The motoneuron counts were therefore made on the entire lumbosacral LMC of the injected side.

Figure 4 shows cross sections of lumbosacral LMCs from control $(A, C)$ and endo $\mathrm{N}$-treated $(B, D)$ embryos at St 33. Sections were sampled from a comparable level of the spinal cord (lumbrosacral segments 4-5). Compared to controls at the same stage, endo $\mathrm{N}$ treatment did not change the overall size and shape of the LMC (Fig. $4 A, B$ ) or the morphology of the
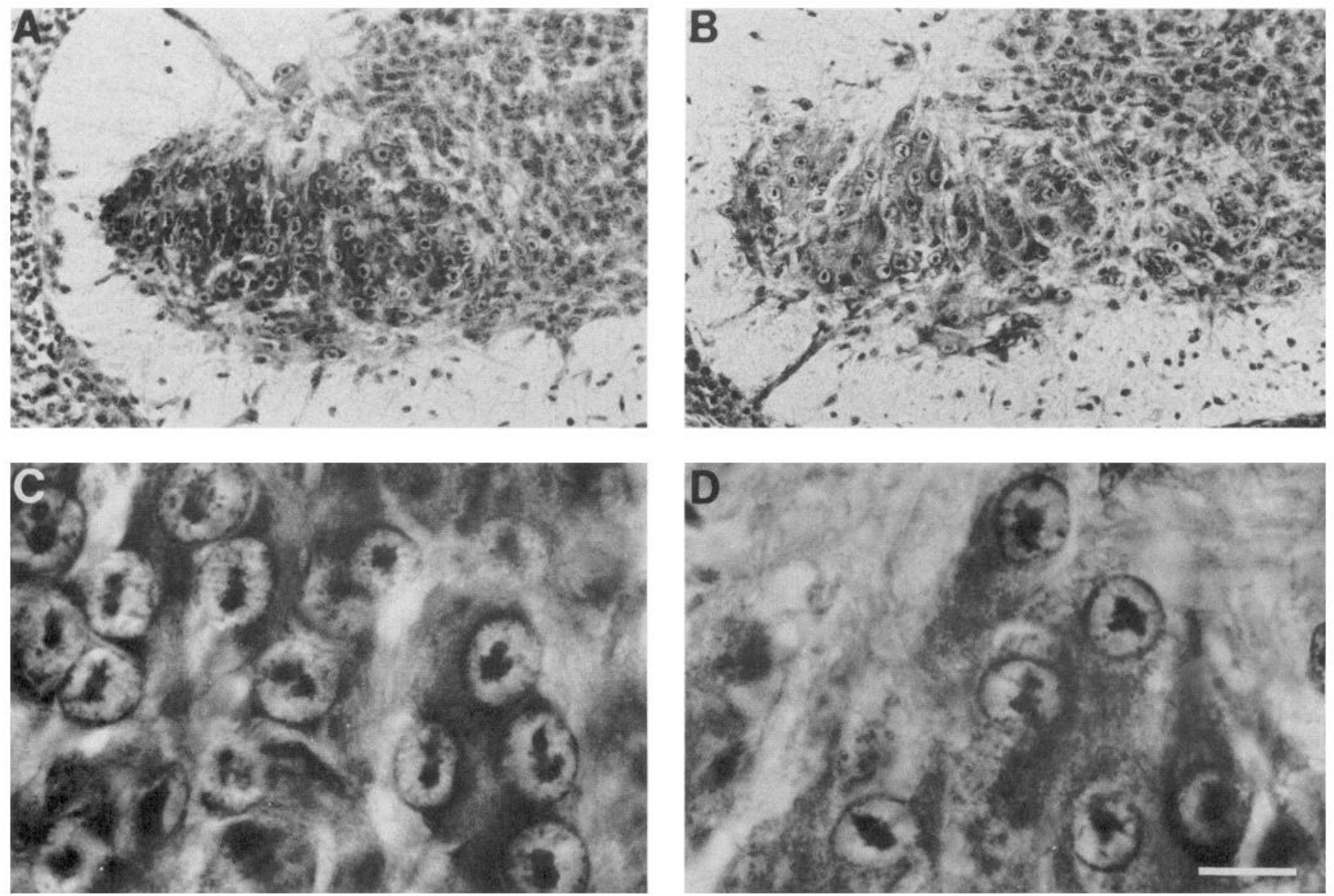

Figure 4. The LMC in control and endo N-treated embryos at St 33: cross sections showing the ventral portion of the spinal cord that contains the LMC at lumbosacral level 4-5 in a control $(A, C)$ and in an endo N-treated embryo $(B, D)$. $C$ and $D$ are enlargements of portions of the LMC from the same sections of $A$ and $B$. Ventral is down, lateral to the left. Scale bar: $50 \mu \mathrm{m}$ for $A$ and $B ; 10 \mu \mathrm{m}$ for $C$ and $D$. 


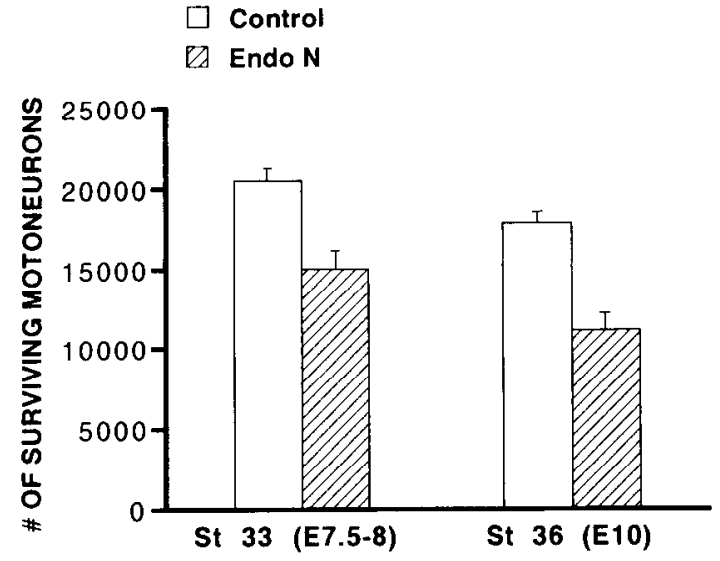

Figure 5. The effect of PSA removal on motoneuron survival: bar graphs showing the total number of surviving lumbosacral motoneurons at St 33, the middle of cell death period, and at St 36, the end of cell death period, in controls (open bars) and in endo $\mathrm{N}$-injected embryos (hatched bars). Error bars $=\mathrm{SD} ; n$ represents the number of embryos; $n=3$ for controls at St 33 and St 36, and for endo N embryos at St 33; $n=5$ for endo $\mathrm{N}$ embryos at St 36 . Differences were significant in both stages $(P \leq 0.01)$. As described in Materials and Methods, these numbers are expressed as raw counts of motoneuron numbers.

motoneurons (Fig. $4 C, D$ ). However, the number of motoneurons was greatly reduced (compare Fig. $4 A, B$ ).

To quantify this difference in lumbosacral motoneuron number, we made counts of the motoneurons from every fifth section in both control and endo N-treated embryos during the cell death period. As shown in Figure 5, following removal of PSA, the number of surviving motoneurons was significantly reduced at both St 33 and St 36. By St 33, the middle of the cell death period, approximately $40-50 \%$ of the motoneurons that will eventually die have already been lost (Hamburger, 1975; Pittman and Oppenheim, 1979; Oppenheim et al., 1992). At this stage there was already a significant decrease in motoneuron survival in the endo $\mathrm{N}$-treated embryos compared to controls; endo $\mathrm{N}$ motoneuron counts were only $73 \%$ of control values (Fig. 5). Between St 33 and St 36 an additional 2655 cells on average were lost in control embryos, while 3929 cells were lost from endo N-treated embryos. Thus, by $\mathrm{St} 36$, which is close to the end of the cell death period, endo N-treated embryos had only $62 \%$ of control motoneuron numbers. Since we determined that the parameter we used for counting (i.e., the nuclear diameter) did not differ between control and endo N-treated embryos, any correction factors for double counting would have reduced the total counts by the same amount for control and experimental animals. Therefore, we did not apply a correction factor and present the data as raw counts (see Materials and Methods for additional details).

Table 1. Raw counts of motoneurons following different treatments

\begin{tabular}{llllll} 
Treatment & $\begin{array}{l}\text { Stage at } \\
\text { injection }\end{array}$ & $\begin{array}{l}\text { Stage at } \\
\text { death }\end{array}$ & $n$ & Results & Ratio \\
\hline Control & - & $36(\mathrm{E} 10)$ & 3 & $17860 \pm 649$ & \\
Endo N & $28(\mathrm{E} 5.5)$ & $36(\mathrm{E} 10)$ & 5 & $11098 \pm 1099$ & 0.6 \\
Boiled endo N & $28(\mathrm{E} 5.5)$ & $36(\mathrm{E} 10)$ & 3 & $16890 \pm 880$ & 0.95 \\
Control & - & $39(\mathrm{E} 13)$ & 3 & $14183 \pm 850$ & \\
Endo N & $37(\mathrm{E} 11)$ & $39(\mathrm{E} 13)$ & 2 & $14530 \pm 820$ & 1.0 \\
\hline
\end{tabular}

Ratio $=$ experimental/control; results are mean \pm SD.
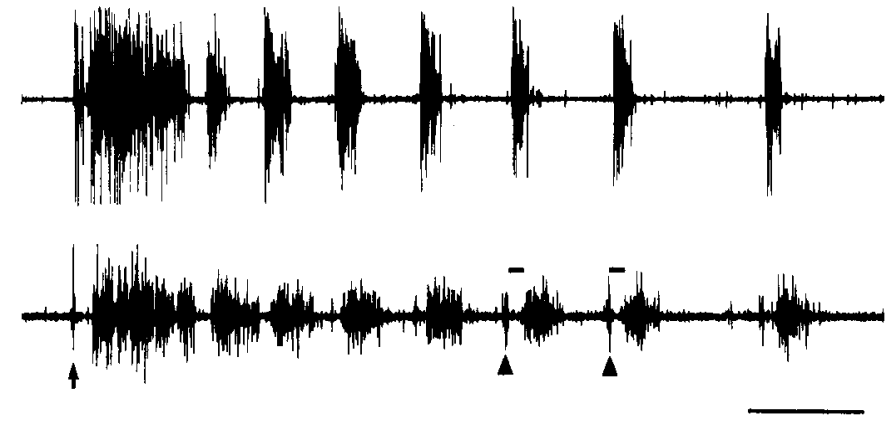

Figure 6. EMG recording from an isolated spinal cord-hindlimb preparation from an St 35 embryo that had been injected with endo $\mathrm{N}$ at St 28. Following a single electrical stimulus to the thoracic cord (indicated by arrow), the PITIB (top trace) and AITIB (bottom trace) muscles exhibited an alternating pattern of activation similar to normal embryos. At the onset of each cycle (several are indicated by arrowheads) the PITIB (an extensor) gives an immediate burst, while the AITIB (a flexor) burst is delayed by an inhibitory period (indicated by the line segments). This results in the alternating pattern, characteristics of these two antagonistic muscles (see Landmesser and O'Donovan, 1984a,b, for additional details). Calibration, $5 \mathrm{sec}$.

To acertain that motoneurons were not killed because of endotoxin toxicity or some other toxic effect of endo $\mathrm{N}$, we carried out several control experiments. First, we injected boiled endo $\mathrm{N}$ (which inactivates endo $\mathrm{N}$ but does not alter endotoxin) at the same stage ( $\mathrm{St} 28$ ) as we did for endo $\mathrm{N}$. In these embryos total motoneuron counts at St 36 did not differ from controls (Table 1). Second, removing PSA at later stages (St 37), after the normal period of nerve branching, synaptogenesis, and motoneuron cell death, also did not affect motoneuron survival (Table 1). Therefore, these data, together with the fact that endo $\mathrm{N}$ did not affect the morphology of surviving motoneurons, indicate that the effects of endo $\mathrm{N}$ we observed on motoneuron survival during the period of cell death are most likely to be a consequence of altered branching and synapse formation rather than any direct or toxic effect of the enzyme on motoneurons. In a previous study, when endo $\mathrm{N}$ was injected at early stages (St 17) and removed PSA during the period of axonal outgrowth, we also did not detect any toxic effects on motoneurons or on any other cells in the spinal cord or limb. This treatment caused some motoneuron projection errors (Landmesser et al., 1990) but did not alter in any detectable way the overall morphology of the spinal cord or hindlimb.

\section{Endo $N$ does not affect motoneuron or muscle activity}

Since the chronic dTC treatment that rescues motoneurons (Pittman and Oppenheim, 1979) also alters their central patterns of activation (Landmesser and Szente, 1986), we wished to determine whether the decreased motoneuron survival caused by endo $\mathrm{N}$ may have resulted indirectly as a consequence of altered motoneuron activity. To test this we examined embryonic motility by counting daily hindlimb movements in ovo and deter-

Table 2. Embryonic motility (hindlimb movements/min)

\begin{tabular}{llll} 
& St 29(E6) & St 31 (E7) & St 33 (E8) \\
\hline Control & $3.5 \pm 0.8$ & $5.7 \pm 0.6$ & $7.7 \pm 0.9$ \\
Endo N & $3.5+0.4$ & $5.8 \pm 0.6$ & $7.6 \pm 0.2$ \\
\hline
\end{tabular}

Mean $\pm \mathrm{SD}, n=3$. 
Table 3. Patterned activity cycles of PITIB and AITIB muscles

\begin{tabular}{lllll} 
& \multicolumn{2}{l}{ Inhibitory period $(\mathrm{sec})$} & \multicolumn{2}{l}{ Burst duration $(\mathrm{sec})$} \\
\cline { 2 - 3 } \cline { 5 - 5 } & Control & Endo N & Control & Endo N \\
\hline PITIB (extensor) & - & - & $1.30 \pm 0.18(25)$ & $1.10 \pm 0.13(6)$ \\
AITIB (flexor) & $0.40 \pm 0.02(9)$ & $0.42 \pm 0.04(6)$ & $1.52 \pm 0.16(9)$ & $1.58 \pm 0.20(6)$ \\
\hline
\end{tabular}

Mean $\pm \mathrm{SD}$ ( $n$, number of bursts).

mined muscle activation patterns by EMG recordings from isolated cord-hindlimb preparations during the period of motoneuron cell death.

Following injection of endo $\mathrm{N}$ at $\mathrm{St} 27$ (E5), motility recordings were collected daily from St 29 (E6) to St 33 (E8). As shown in Table 2, no significant differences in motility levels between endo $\mathrm{N}$-treated and control embryos were found at any time during the three day recording period.

Muscle activation patterns were examined at later stages (St 34-36, E8-E10) from some of the same embryos used for motility recordings. In general, the various muscles we recorded from in endo $\mathrm{N}$-treated embryos, two flexors [sartorius and anterior iliotibialis (AITIB)] and two extensors (femorotibialis and PITIB), exhibited activation patterns that were indistinguishable from those obtained previously in normal embryos (Landmesser and O'Donovan, 1984a,b). For example, as shown in Figure 6 , a single electrical stimulus (indicated by arrow in Fig. 6) to the thoracic cord initiated a series of bursts (seven in this case) from the pair of recorded muscles (PITIB on the top and AITIB on the bottom). These muscles, which are functional antagonists, exhibited a pattern of alternating bursts. The mean burst durations and the duration of the inhibitory period in the AITIB burst in endo $\mathrm{N}$-treated embryos did not differ significantly from control values (Table 3 ). Therefore, we conclude that endo $\mathrm{N}$ treatment does not alter in any appreciable way the activity patterns of motoneurons or muscles.

\section{Discussion}

The data presented in this article provide a clear correlation between the ability of motoneurons to survive the cell death period and the extent to which they branch within muscles and form synapses. The reduction in neuronal branching produced by endo $\mathrm{N}$ resulted in a reduction in the number of synapses, which in turn was correlated with decreased motoneuron survival. Since the number of synapses was already decreased to about half of control values at the onset of the motoneuron cell death period (St 30), the change in synapse number cannot be due to increased death of motoneurons (which only begins at St 30), but may in fact be the cause of the increased cell death. These results therefore support the "access" hypothesis, in other words, that regulation of nerve branching and synapse formation can determine the extent to which developing motoneurons obtain trophic support from their target during the period of naturally occurring cell death (Meriney and Pilar, 1987; Dahm and Landmcsscr, 1988; Oppenheim, 1989, 1991).

Previous reports have shown that blockade of neuromuscular activity in chick embryos increases intramuscular nerve branching, synaptogenesis (Dahm and Landmesser, 1988, 1991), and motoneuron survival (Pittman and Oppenheim, 1978, 1979). These results suggested that changes in nerve branching and synapse number following activity blockade might be a cause of the ubserved increased motoneuron survival. However, activity blockade may alter motoneuron survival by affecting other events. For example, both dTC and $\alpha$-BTX have been shown to reduce the normal activation of motoneurons during spontaneous motility (Landmesser and Szente, 1986) in addition to blocking the neuromuscular junction. Such a reduction of motoneuron activation might reduce intracellular free $\mathrm{Ca}^{2+}$, and thereby interfere with the triggering of cell death at a point downstream from where trophic factors are acting. It has in fact recently been found that nicotinic agonist binding to $\alpha$-BTXbinding sites on ciliary neurons results in an increase in intracellular free $\mathrm{Ca}^{2+}$ (Vijayaraghaven et al., 1992) and dTC or $\alpha$-BTX could prevent this. It is also possible that less active motoneurons would require smaller amounts of trophic factor for survival. Thus, these earlier experiments (Pittman and Oppenheim, 1978, 1979; Dahm and Landmesser, 1988, 1991), while suggestive, could not provide a direct link between branching, synapse formation, and motoneuron survival. The present results provide stronger support for the idea that the extent of intramuscular nerve branching and synaptogenesis is an important variable in the regulation of motoneuron cell death.

The only obvious effect of PSA removal during the developmental stages studied was a reduction in intramuscular nerve branching and, what we believe is a secondary consequence of this, a reduction in the number of synapses formed. We did not detect any other anatomical or physiological consequences of PSA removal on either nerve or muscle development (including the number of muscle fibers formed; B. Fredette, unpublished observations). While we cannot completely exclude that PSA removal is affecting motoneuron cell death by some, as yet unspecificd, effect on motoneurons, we believe, as detailed below, that our data are best explained by the effects of PSA on intramuscular nerve branching.

The previously described increase in intramuscular branching induced by activity blockade was found to be associated with an increase in axonal PSA levels. Furthermore, removal of PSA was found to prevent these inactivity-induced effects on branching (Landmesser et al., 1990). In addition injection of endo $\mathrm{N}$ into normally active embryos itself reduced intramuscular nerve branching (Landmesser et al., 1988), although neither synapse number nor motoneuron survival was quantified in that study. Since the effects of PSA on intramuscular nerve branching are most simply explained by it modulating the function of adhesion molecules on the growing nerve and muscle (Landmesser et al., 1988, 1990) as it does in other systems (Rutishauser et al., 1988; Acheson et al., 1991; Tang et al., 1992; Landmesser, 1993), alteration of PSA levels by endo $\mathrm{N}$ injections provided us with another (activity-independent) way of altering branching and synapse number. We found that removal of PSA decreased nerve branching, synaptogenesis, and motoneuron survival without affecting motoneuron or muscle activity, and therefore we favor the straightforward explanation that endo $\mathrm{N}$ treatment decreased access of motoneurons to muscle-derived trophic factors by decreasing intramuscular nerve branching and synaptic sites, and that this increased motoneuron death. 
Although NCAM carries the vast majority of PSA in our system (Fredette et al., 1992; Tang et al., 1992), injection of endo $\mathrm{N}$ would also remove PSA from any other molecules that contained PSA of the form recognized by the enzyme (i.e., $\alpha-2,8-$ linked PSA of chain length greater than five sialyl residues; Vimr et al., 1984; Rutishauser et al., 1985; Acheson et al., 1991). PSA of this type has been observed on sodium channels of the eel electroplax (James and Agnew, 1987), and while we did not monitor this, if sodium channels in our system and at this developmental stage contain PSA, this also would have been removed by our endo $\mathrm{N}$ injections. While some effect of this on the events we have described in this article (i.e., nerve branching, synapse formation, and motoneuron survival) cannot be completely discounted, several observations lead us to conclude that PSA modulation of sodium channel function is unlikely to explain our results.

First, although removal of PSA was found to affect the gating properties of the sodium channel (Recio-Pinto et al., 1990) this only occurred after prolonged $(5 \mathrm{hr})$ treatment with neuraminidase, which would have removed virtually all of the sialic acid residues. The authors in fact suggested that it was only removal of the sialic acid residues closest to the membrane that contributed to the alteration in gating properties. In contrast to the exo-neuraminidase used in the previous studies (Recio-Pinto et al., 1990), the endo $\mathrm{N}$ used in our experiments cleaves longchain-length PSA, but leaves five sialic acid residues intact, and this probably would have been sufficient to maintain normal gating properties. Second, we detected no changes in the amount or pattern of muscle activity. The normal EMGs that we recorded indicate that motoneurons were activated by spinal circuits in a normal manner, that those patterns of activity were conducted normally to the nerve ending, and that this resulted in appropriate activation of the muscle fibers. Thus, any alteration in sodium channel function, if it indeed occurred, had no effect at this level of analysis. Finally, PSA removal at later stages, when intramuscular nerve branching and synaptogenesis were essentially complete, would presumably have affected sodium channel function in a similar manner, but this had no effect on motoneuron survival. Thus, we believe our results are best explained by PSA modulation of adhesion molecule function, which in turn affects intramuscular nerve branching, synaptogenesis, and motoneuron survival.

I hus far, motoneuron survival has shown a good correlation with alterations in the extent of intramuscular nerve branching and synapse number produced either by activity blockade (Dahm and Landmesser, 1988, 1991) or by endo $\mathrm{N}$ injections (present report). Clearly, the access hypothesis that we and others have proposed (Meriney and Pilar, 1987; Dahm and Landmesser, 1988, 1991; Oppenheim, 1989, 1991) will be strengthened if such a correlation is found to hold when branching and synaptogenesis are altered by additional means. Injections of antiNCAM were shown to decrease intramuscular nerve branching, while anti-L1 increased it (Landmesser et al., 1988). Thus, while technically difficult for reasons enumerated in the introductory remarks, determination of synapse number and motoneuron survival during the cell death period in such preparations would provide additional means for testing the access hypothesis.

However it is regulated, the actual role played by naturally occurring neuronal cell death, including that of motoneurons, is still under debate. Several studies have provided strong suggestive cvidence that one role may be to match the size of neuronal populations and their targets (Tanaka and Landmes- ser, 1986; Sperry, 1987; Vogel et al., 1989). While certain observations do not fit easily into a simple, linear size-matching scheme (Lamb, 1980; Sperry, 1987; for review, see Oppenheim, 1991), they are not inconsistent with more complex versions in which both anterograde and retrograde trophic interactions occur between neurons and their targets (Galli-Resta et al., 1992) and in which activity may serve to modulate these influences. A more complete understanding of the exact cellular mechanisms by which neurons and their targets interact during this critical period should help in assessing the validity of the trophic hypothesis (Purves, 1988) as related to naturally occurring cell death in this system.

\section{References}

Abercrombie M (1946) Estimation of nuclear population from microtome sections. Anat Rec 94:236-247.

Acheson A, Sunshine JL, Rutishauser U (1991) NCAM polysialic acid can regulate both cell-cell and cell-substrate interactions. J Cell Biol 114:143-153.

Buckley K, Kelly R (1985) Identification of a transmembrane glycoprotein specific for secretory vesicles of neuronal and endocrine cells. J Cell Biol 100:1284-1294.

Chuong CM, Edelman GM (1984) Alterations in neural cell adhesion molecules during development of different regions of the nervous system. J Neurosci 4:2354-2366.

Dahm L, Landmesser L (1988) The regulation of intramuscular nerve branching during normal development and following activity blockade. Dev Biol 130:621-644.

Dahm L, Landmesser L (1991) The regulation of synaptogenesis during normal development and following activity blockade. J Neurosci 11:238-255.

Ding R, Jansen JKS, Laing NG, Tonnesen H (1983) The innervation of skeletal muscle in chickens curarized during early development. J Neurocytol 12:887-919.

Doherty P, James C, Walsh FS (1990) Neurite outgrowth in response to transfected N-CAM changes during development and is modulated by polysialic acid. Neuron 5:209-219.

Fredette BJ, Landmesser LT, Rustishauser U (1992) Distribution of NCAM isoforms and N-cadherin on developing muscle cell surfaces. Soc Neurosci Abstr 18:1325.

Galli-Resta L, Resta G (1992) A quantitative model for the regulation of naturally occurring cell death in the developing vertebrate nervous system. J Neurosci 12:4586-4594.

Hamburger V (1975) Cell death in the development of the lateral motor column of the chick embryo. J Comp Neurol 160:535-546.

Hamburger V, Hamilton HL (1951) A series of normal stages in the development of the chick embryo. J Morphol 88:49-82.

Henderson CE, Huchet M, Changeux JP (1983) Denervation increases a neurite-promoting activity in extracts of skeletal muscle. Nature 302:609-611.

Hoffman S, Edelman GM (1983) Kinetics of homophilic binding by embryonic and adult forms of the neural cell adhesion molecule. Proc Natl Acad Sci USA 80:5761-5766.

Hill MA, Bennett MD (1983) Cholinergic growth factor from skeletal muscle is elevated following denervation. Neurosci Lett 35:31-36.

James WM, Agnew WS (1987) Multiple oligosaccharide chains in the voltage-sensitive Na channel from Electrophorus electricus: evidence for alpha-2,8-linked polysialic acid. Biochem Biophys Res Commun 148:817-826.

Lamb A (1980) Motoneuron counts in Xenopus frogs reared with one bilaterally innervated hindlimb. Nature 284:347-350.

Landmesser $L$ (1993) The effects of in-vivo alteration of polysialic acid on avian nerve-muscle development. In: Polysialic acid (Roth J, ed), pp 229-239. Boston: Birkhauser.

Landmesser L, O'Donovan M (1984a) Activation patterns of embryonic chick hindlimb muscles recorded in ovo and in an isolated spinal cord preparation. J Physiol (Lond) 347:189-204.

Landmesser L, O'Donovan M (1984b) The activation patterns of embryonic chick motoneurons projecting to inappropriate muscles. J Physiol (Lond) 347:205-224.

Landmesser L, Szente M (1986) Activation patterns of embryonic 
chick hindlimb muscle following blockade of activity and motoneuron cell death. J Physiol (Lond) 380:157-174.

Landmesser L, Dahm L, Schultz K, Rutishauser U (1988) Distinct roles of adhesion molecules during innervation of embryonic chick muscle. Dev Biol 130:645-670.

Landmesser L, Dahm L, Tang J, Rutishauser U (1990) Polysialic acid as a regulator of intramuscular nerve branching during embryonic development. Neuron 4:655-667.

Meriney SD, Pilar GP (1987) Cholinergic innervation of the smooth muscle cells in the choroid coat of the chick cyc and its development. J Neurosci 7:3827-3839.

Oppenheim RW (1989) The neurotrophic theory and naturally occurring motoneuron death. Trends Neurosci 12:252-255.

Oppenheim RW (1991) Cell death during development of the nervous system. Annu Rev Neurosci 14:453-501.

Oppenheim RW, Chu-Wang I-w (1983) Aspects of naturally-occurring motoneuron death in the chick spinal cord during embryonic development. In: Somatic and autonomic nerve-muscle interactions (Burnstock G, ed), pp 57-107. Amsterdam: Elsevier.

Oppenheim RW, Qin-Wei Y, Prevette D, Yan Q (1992) Brain-derived neurotrophic factor rescues developing avian motoneurons from cell death. Nature 360:765-757.

Pittman R, Oppenheim RW (1978) Neuromuscularblockade increases motoneuron survival during normal cell death in the chick embryo. Nature 271:364-366.

Pittman R, Oppenheim RW (1979) Cell death of motoneurons in the chick embryo spinal cord. IV. Evidence that a functional neuromuscular interaction is involved in the regulation of naturally occurring cell death and the stabilization of synapses. J Comp Neurol 187:425446.

Purves D (1988) Body and brain, a trophic theory of neuronal connections. Cambridge, MA: Harvard UP.

Recio-Pinto E, Thornhill WB, Duch DS, Levinson SR, Urban BW (1990) Neuraminidase treatment modifies the function of electroplax sodium channels in planar lipid bilayers. Neuron 5:675-684.
Rutishauser U, Watanabe M, Silver J, Troy FA, Vimr ER (1985) Specific alteration of NCAM-mediated cell adhesion by an endoneuraminirlase. J Cell Biol 101:1842-1849.

Rutishauser U, Acheson A, Hall AK, Mann DM, Sunshine J (1988) The neural cell adhesion molecule (NCAM) as a regulator of cell-cell interactions. Science 240:53-57.

Sperry DG (1987) Relationship between natural variations in motoneuron number and body size in Xenopus laevis: a test for size matching. J Comp Neurol 264:250-267.

Sunshine J, Balak K, Rutishauser U, Jacobson M (1987) Changes in neural cell adhesion molecule (NCAM) structure during vertebrate neural development. Proc Natl Acad Sci USA 84:5986-5990.

Tanaka H (1987) Chronic application of curare does not increase the level of motoneuron survival-promoting activity in limb muscle extracts during the naturally occurring motoneuron cell death period. Dev Biol 124:347-357.

Tanaka H, Landmesser L (1986) Cell death of lumbosacral motoneurons in chick, quail, and chick-quail chimeras: a test of the quantitative matching hypothesis of cell death. J Neurosci 6:2889-2899.

Tang J, Landmesser L (1992) Reduction of intramuscular nerve branching results in decreased motoneuron survival. Soc Neurosci Abstr 18:45.

Tang J, Landmesser L, Rutishauser U (1992) Polysialic acid influences specific pathfinding by avian motoneurons. Neuron 8:1031-1044.

Vijayaraghaven S, Pugh PC, Zhang Z-w, Rathouz MM, Berg DK (1992) Nicotinic receptors that bind $\alpha$-bungarotoxin on neurons raise intracellular free- $\mathrm{Ca}^{++}$. Neuron 8:353-362.

Vimr ER, McCoy RD, Vollger HF, Wilkison NC, Troy FA (1984) Use of prokaryotic-derived probes to identify poly(sialic acid) in neonatal neural membranes. Proc Natl Acad Sci USA 81:1971-1975.

Vogel MW, Sunter K, Herrup K (1989) Numerical matching between granule and Purkinje cells in lurcher chimeric mice: a hypothesis for the trophic rescue of granule cells from target-related cell death. J Neurosci 9:3454-3462. 